\title{
Study of $K^{-} \rightarrow \pi^{0} e^{-} \bar{v}_{e} \gamma$ decay with ISTRA+ setup.
}

\author{
V.N. Bolotov* \\ Institute for Nuclear Research, Moscow, Russia \\ E-mail:bolotoveinr.ru, bolotovvnemail.ru \\ E.N. Guschin, V.A. Duk, S.V. Laptev, V.A. Lebedev, A.E. Mazurov, A.Yu. \\ Polyarush,V.E. Postoev \\ Institute for Nuclear Research, Moscow, Russia.
}

S.A. Akimenko, G.I. Britvich, K.V. Datsko, A.P. Filin, A.V. Inyakin, V.F.Konstantinov, A.S.Konstantinov, I.Y. Korolkov, V.A.Khmelnikov, V.M. Leontiev, V.P. Novikov, V.F.Obraztsov, V.A.Polyakov, V.I. Romanovsky, V.I. Shelikhov, O.G. Tchikilev, V.A. Uvarov, O.P. Yushchenko

Institute for High Energy Physics, Protvino, Russia.

Results of the study of the $K^{-} \rightarrow \pi^{0} e^{-} \bar{v} \gamma$ decay at ISTRA+ setup are presented. 4476 events of this decay have been observed. The ratio of branching ratios (R), $R=\frac{B r\left(K^{-} \rightarrow \pi^{0} e^{-} \bar{v}_{e} \gamma\right)}{\operatorname{Br}\left(K^{-} \rightarrow \pi^{0} e^{-} \bar{v}_{e}\right)}=$ $(1.81 \pm 0.03$ (stat. $) \pm 0.07$ (syst. $)) \times 10^{-2}$, has been obtained for $E_{\gamma}^{*}>10 \mathrm{MeV}$ and $\theta_{e \gamma}^{*}>10^{\circ}$. For comparison with previous experiments, the branching ratio with cuts $E_{\gamma}^{*}>10 \mathrm{MeV}, 0.6<$ $\cos \theta_{e \gamma}^{*}<0.9$ is calculated, $R=\frac{B r\left(K^{-} \rightarrow \pi^{0} e^{-} \bar{v}_{e} \gamma\right)}{B r\left(K^{-} \rightarrow \pi^{0} e^{-} \bar{v}_{e}\right)}=(0.47 \pm 0.02($ stat. $) \pm 0.03($ syst. $)) \times 10^{-2}$. For the cuts $E^{*}(\gamma)>30 \mathrm{MeV}$ and $\theta_{e \gamma}^{*}>20^{\circ}$, used in most theoretical papers, we have obtained $\operatorname{Br}\left(K^{-} \rightarrow \pi^{0} e^{-} \bar{v}_{e} \gamma\right)=(3.13 \pm 0.09 \pm 0.14) \times 10^{-4}$. For the asymmetry $A_{\xi}$ we get $A_{\xi}=-0.015 \pm 0.021$. At present it is the best estimate of this asymmetry.

KAON International Conference

May 21-25 2007

Laboratori Nazionali di Frascati dell'INFN, Rome, Italy

\footnotetext{
* Speaker.
} 


\section{INTRODUCTION}

The decay $K^{-} \rightarrow \pi^{0} e^{-} \bar{v} \gamma$ provides fertile testing ground for the Chiral Theory (ChPT) [1, 2]. $K^{-} \rightarrow \pi^{0} e^{-\bar{v}} \gamma$ decay amplitudes are calculated at order $O\left(p^{4}\right)$ in [1], and branching ratios are evaluated in [3]. Recently, next-to-leading $O\left(p^{6}\right)$ corrections were calculated for the corresponding neutral kaon decay [4].

The matrix element for $K^{-} \rightarrow \pi^{0} e^{-} \bar{v} \gamma$ has general structure

$$
\begin{gathered}
T=\frac{G_{F}}{\sqrt{2}} e V_{u s} \varepsilon^{\mu}(q)\left\{\left(V_{\mu v}-A_{\mu v}\right) \bar{u}\left(p_{v}\right) \gamma^{v}\left(1-\gamma_{5}\right) v\left(p_{l}\right)\right. \\
\left.+\frac{F_{v}}{2 p_{l} q} \bar{u}\left(p_{v}\right) \gamma^{v}\left(1-\gamma_{5}\right)\left(m_{l}-\hat{p}_{l}-\hat{q}\right) \gamma_{\mu} v\left(p_{l}\right)\right\} \equiv \varepsilon^{\mu} A_{\mu} .
\end{gathered}
$$

First term of the matrix element describes Bremsstrahlung of kaon and direct emission (Fig. 1a). The lepton Bremsstrahlung is presented by the second term in r.h.s Eq.(1) and Fig. $1 b$.

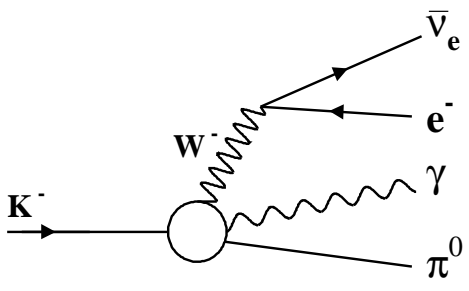

a)

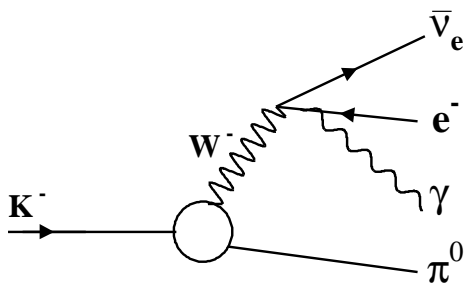

b)

Figure 1: Diagrammatic representation of the $K_{l 3 \gamma}$ amplitude.

The $K^{-} \rightarrow \pi^{0} e^{-} \bar{v} \gamma$ decay is one of kaon decays where new physics beyond the SM can be probed. This decay is especially interesting as it is sensitive to $T$-odd contributions. According to CPT theorem, observation of $T$ violation is equivalent to observation of $C P$-violating effects.

In the SM the source of $C P$ violation is given by the phase in the CKM matrix [5, 6, 7]. However, it has been argued that this source is not enough to explain the observed baryon asymmetry of the Universe and new sources of $C P$ violation have to be introduced [8].

Important experimental observable used in $C P$ violation searches is the $T$-odd correlation, for $K^{-} \rightarrow \pi^{0} e^{-} \bar{v} \gamma$ decay defined as

$$
\xi=\frac{1}{M_{K}^{3}} p_{\gamma} \cdot\left[p_{\pi} \times p_{e}\right] .
$$

First suggestion to investigate $T$-odd triple-product correlations was done in [9].

To establish the presence of a nonzero triple-product correlation, one constructs a $T$-odd asymmetry of the form 


$$
A_{\xi}=\frac{N_{+}-N_{-}}{N_{+}+N_{-}}
$$

where $N_{+}$and $N_{-}$are numbers of events with $\xi>0$ and $\xi<0$.

$T$-odd correlation vanishes at tree level in the SM [11], but the SUSY theory gives rise to $C P$-odd $(T-$ odd $)$ observables already at tree level [12, 13, 14]. $T$-odd asymmetry value for $S U(2)_{L} \times S U(2)_{R} \times U(1)$ model and scalar models was estimated in [15].

In this paper we present first results of the analysis of the $K^{-} \rightarrow \pi^{0} e^{-} \bar{v} \gamma$ data accumulated by ISTRA+ experiment during the 2001 run.

\section{ISTRA+ SETUP}

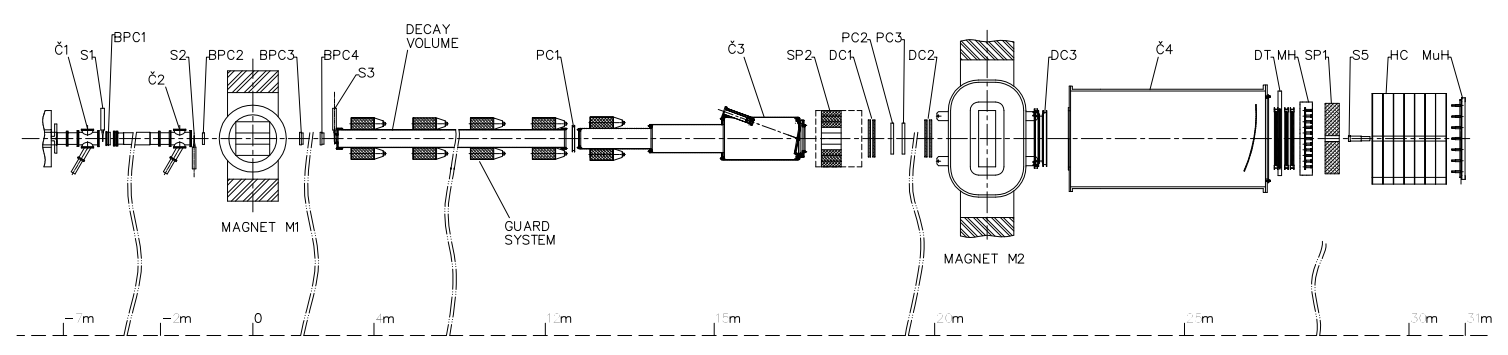

Figure 2: View of the ISTRA+ detector.

The experiment was performed using ISTRA+ detector which is modernized ISTRA-M detector [16]. ISTRA+ detector (see Fig. 2) is located at a negative unseparated beam. The measurement of the beam particles, deflected by the beam magnet $M 1$ is performed by four beam chambers $B P C_{1} \div B P C_{4}$. The beam momentum is $\sim 25 \mathrm{GeV}$ with $\Delta p / p \sim 1.5 \%$. Admixture of $K^{-}$in the beam is $\sim 3 \%$. The beam intensity is $\sim 3 \times 10^{6}$ per $1.9 \mathrm{~s} \mathrm{U}-70$ spill. The kaon identification is performed by $\check{C}_{0}-\check{C}_{2}$ threshold $\check{C}$ counters $\left(\check{C}_{0}\right.$ is not shown in Fig. 2$)$.

The decay products are deflected by the spectrometer magnet $M 2$ with the field integral of 1 $\mathrm{Tm}$. The track measurement is performed by $1-\mathrm{mm}-$ step proportional chambers $\left(P C_{1} \div P C_{3}\right), 2-$ cm-cell drift chambers $\left(D C_{1} \div D C_{3}\right)$, and by four planes of the $2-\mathrm{cm}$-diameter drift tubes DT. The photons are measured by lead-glass electromagnetic calorimeter $S P_{1}$ consisting of 576 counters. The counter transverse size is $5.2 \times 5.2 \mathrm{~cm}$ and length is about $15 X_{0}$. Decay volume is surrounded by eight lead-glass rings to veto low energy photons. Lead-glass electromagnetic calorimeter $\mathrm{SP}_{2}$ is also used as a part of the veto system.

\section{EVENT SELECTION}

During physics run in November-December 2001, 350M events were logged on tapes. This information is complemented by $260 \mathrm{M}$ events generated with Geant3 [17]. The Monte-Carlo simulation includes a realistic description of the experimental setup: the decay volume entrance windows, the track chamber windows, gas mixtures, sense wires and cathode structures, Cherenkov counter mirrors and gas mixtures, the shower development in the electromagnetic calorimeters, 
etc. The detailed discussion of the simulation and reconstruction procedure is given in our previous publications [18, 19].

Events with one negative track detected in the tracking system and four showers detected in the electromagnetic calorimeter $S P_{1}$ are selected as candidates for $K^{-} \rightarrow \pi^{0} e^{-} v \gamma$ decay. One of the showers must be associated with the charged track. Events inside interval $400<z<1650 \mathrm{~cm}$, and transverse radius less than $10 \mathrm{~cm}$ are selected for further analysis. The probability of the vertex fit, C.L. $\left(\chi^{2}\right)$, is required to be more than $10^{-4}$. Absence of signals in veto system above noise threshold is required. The electron identification is done using the $E / p$ ratio of the energy of the cluster associated with the track to the momentum of this track given by tracking system. This ratio must be inside interval 0.80-1.15 (see Fig. 3). Another cut used for the suppression of the $\pi^{-}$contamination is that on the distance between the charged track extrapolation to the front plane of the electromagnetic detector and the nearest shower. This distance must be less than $2.5 \mathrm{~cm}$. The effective mass $m_{\gamma \gamma}$ within $30 \mathrm{MeV}$ from $\pi^{0}$ table mass (Fig. 4) is required. At the end, the convergence of the $2 \mathrm{C} K^{-} \rightarrow \pi^{0} e^{-} \bar{v}_{e} \gamma$ kinematic fit is required.

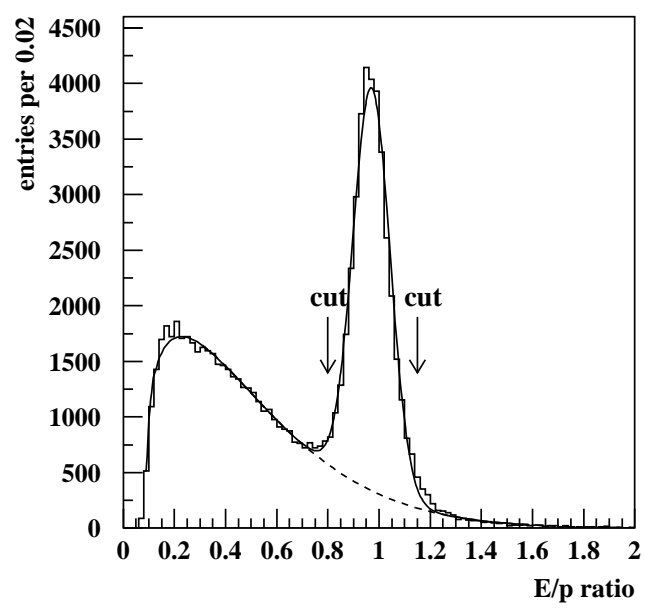

Figure 3: E/P ratio for the real data. Dotted line is our fit of background.

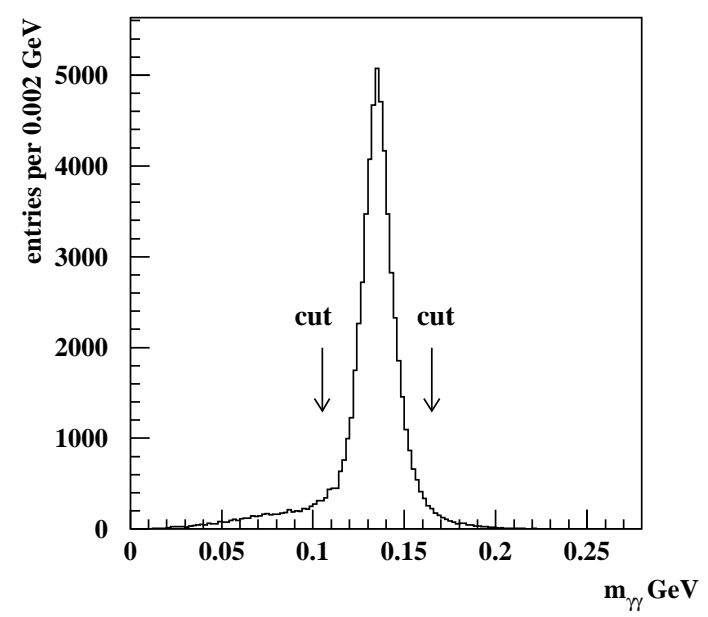

Figure 4: $\gamma \gamma$ mass for the real data.

\section{BACKGROUND SUPPRESSION}

The main background channels for the decay $K^{-} \rightarrow \pi^{0} e^{-} \bar{v}_{e} \gamma$ are:

(1) $K^{-} \rightarrow \pi^{-} \pi^{0} \pi^{0}$ where one of the $\pi^{0}$ photons is not detected and $\pi^{-}$decays to $e v$ or is misidentified as an electron.

(2) $K^{-} \rightarrow \pi^{-} \pi^{0}$ with "fake photon" and $\pi^{-}$decayed or misidentified as electron. Fake photon clusters can come from $\pi \mathrm{n}$ interaction in the detector, external bremsstrahlung upstream of the magnet, accidentals. All these sources are included in our MC calculations.

(3) $K^{-} \rightarrow \pi^{0} e^{-} \bar{v}$ with extra photon. The main source of extra photons is an electron interaction in the detector. 

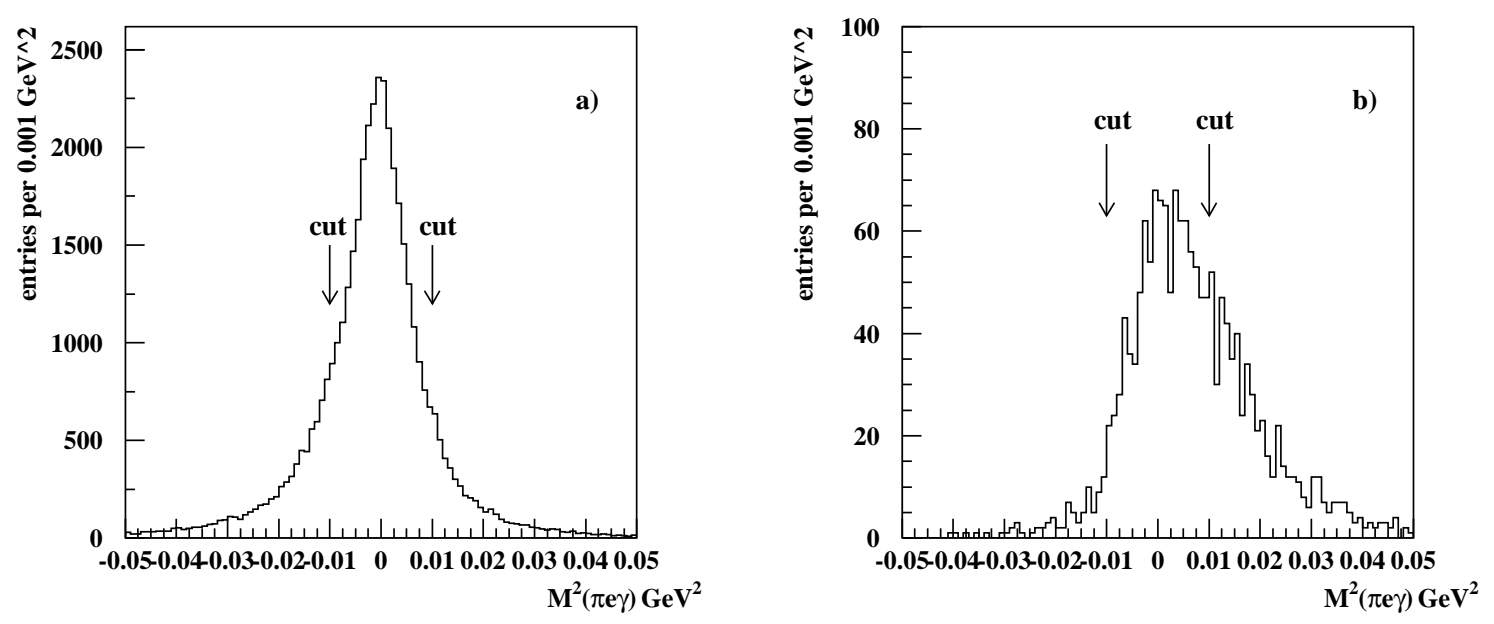

Figure 5: Missing mass $M^{2}\left(\pi^{0} e^{-} \gamma\right)$ distribution: a) for the real data; b) for the background at point (1).

\begin{tabular}{|c|c|c|c|}
\hline Cut & Real data & Background & Signal \\
\hline Without cuts & 41072 & 32901 & 11180 \\
$E_{\text {miss }}>0.5 \mathrm{GeV}$ & 37428 & 31134 & 10035 \\
$-0.01<M^{2}\left(\pi^{0} e^{-} \gamma\right)<0.01 \mathrm{GeV}^{2}$ & 26277 & 25287 & 8430 \\
$M^{2}\left(\pi^{-} \pi^{0}\right)<0.009 \mathrm{GeV}^{2}$, & 23293 & 21648 & 7153 \\
$M^{2}\left(\pi^{-} \pi^{0}\right)>0.024 \mathrm{GeV}^{2}$ & & & \\
$0.002<\theta_{e \gamma}<0.030$ & 6079 & 1603 & 4476 \\
\hline
\end{tabular}

Table 1: Event reduction statistics for the real data, the MC background and the signal.

(4) $K^{-} \rightarrow \pi^{-} \pi^{0} \gamma$ when $\pi^{-}$decays or is misidentified as electron.

(5) $K^{-} \rightarrow \pi^{0} \pi^{0} e^{-} v$ when one $\gamma$ is lost.

From Fig. 3 it is seen that in raw data background contamination from channels with charged pion in final state is about $15 \%$. Requirement on the missing energy $E_{\text {miss }}>0.5 \mathrm{GeV}$ in the decay reduces mainly background channel at point (4). For the suppression of the background channels $(1 \div 5)$ we use a cut on the missing mass squared $M^{2}\left(\pi^{0} e \gamma\right)=\left(P_{K}-P_{\pi^{0}}-P_{e}-P_{\gamma}\right)^{2}$. For the signal events this variable corresponds to the square of the neutrino mass and must be zero within measurement accuracy (see Fig. 5); we require $-0.01<M^{2}\left(\pi^{0} e^{-} \gamma\right)<0.01 \mathrm{GeV}^{2}$, as shown in table 1. For the suppression of the background channel(1) we also use a cut on the missing mass squared $M^{2}\left(\pi^{-} \pi^{0}\right)=\left(P_{K}-P_{\pi^{-}}-P_{\pi^{0}}\right)^{2}$. For the events of the background channel (1) this variable corresponds to the $\pi^{0}$ mass, while the distribution of this variable for the signal is rather wide (see Fig. 6); we select events with $M^{2}\left(\pi^{-} \pi^{0}\right)<0.009 \mathrm{GeV}^{2}, M^{2}\left(\pi^{-} \pi^{0}\right)>0.024 \mathrm{GeV}^{2}$.

The dominant background channel to $K_{e 3 \gamma}$ arises from $K_{e 3}$ with extra photon. The background channel (3) is suppressed by requirement on the angle between electron and photon in the laboratory frame, $\theta_{e \gamma}, 0.002<\theta_{e \gamma}<0.030$. (see Fig. 7). The distribution of the $K_{e 3}$-background events 
has a very sharp peak at zero angle. This peak is significantly narrower than that for signal events. This happens, in particular, because the emission of the photons by the electron from $K_{e 3}$ decay occurs in the setup material downstream the decay vertex, but angle is still calculated as if emission comes from the vertex. Right part of this cut is introduced for suppression of background channels $(1,2,4,5)$.

After all cuts 6079 events are selected, with a background of 1603 events. Background normalization was done by comparison numbers of events for $K_{e 3}$ decay in $\mathrm{MC}$ and real data samples. Event reduction statistics is summarized in Table 1.
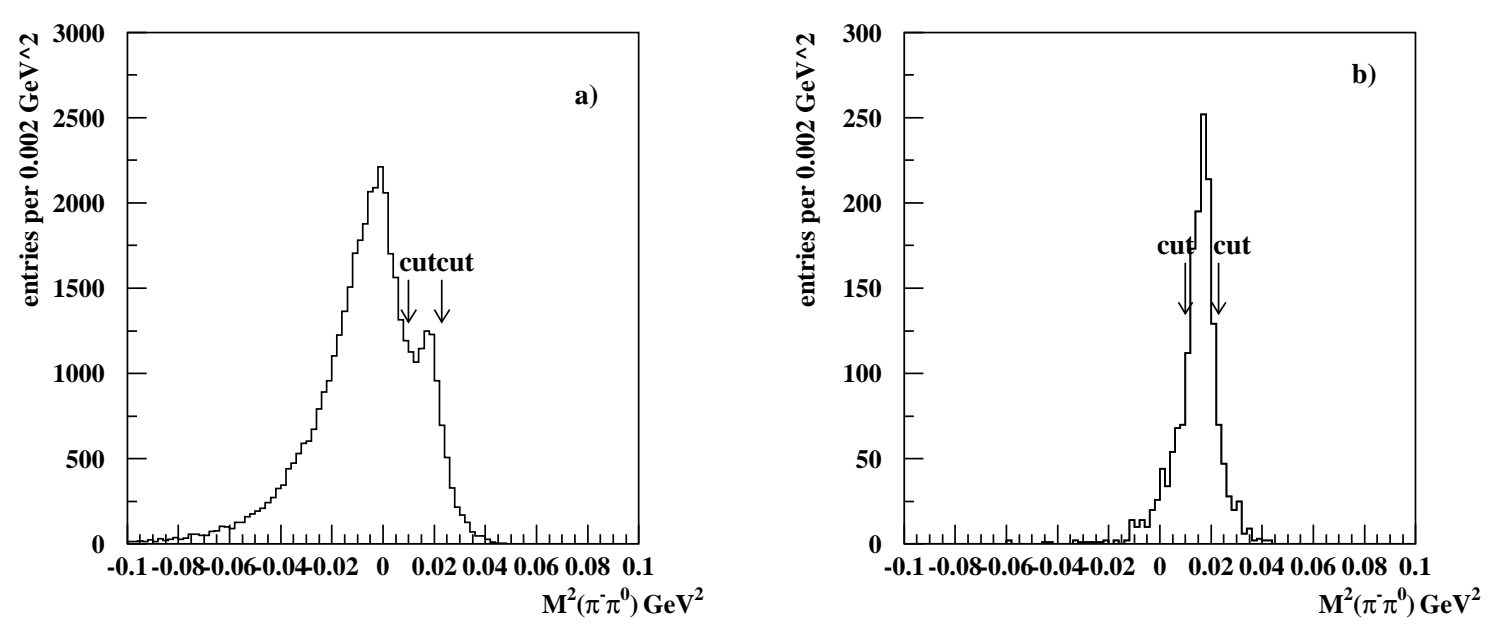

Figure 6: Missing mass distribution $M^{2}\left(\pi^{-} \pi^{0}\right)$ : a) for real data; b) for the background at point (1).

\section{RESULTS}

The resulting distribution of the selected events over $\cos \left(\theta_{e \gamma}^{*}\right), \theta_{e \gamma}^{*}$ being the angle between the electron and the photon in the kaon rest frame, is shown in Fig.8. The distribution over $E_{e \gamma}^{*}$ - the photon energy in the kaon rest frame - is shown in Fig. 9. Reasonable agreement of the data with $\mathrm{MC}$ is seen. Generator based on $O\left(p^{2}\right)$ ChPT [11] is used.

To obtain the branching ratio of the $K_{\pi^{0} e^{-} \bar{v}_{e} \gamma}$ relative to the $K_{e 3}(\mathrm{R})$, the background and efficiency corrected number of $K_{e 3 \gamma}$ events is compared to $569923 K_{e 3}$ events found with similar selection criteria. The ratio of branching ratio $(\mathrm{R})$ is found to be:

$$
R=\frac{B r\left(K^{-} \rightarrow \pi^{0} e^{-} \bar{v}_{e} \gamma\right)}{B r\left(K^{-} \rightarrow \pi^{0} e^{-} \bar{v}_{e}\right)}=(1.81 \pm 0.03(\text { stat. }) \pm 0.07(\text { syst. })) \times 10^{-2}
$$

for $E_{\gamma}^{*}>10 \mathrm{MeV}$ and $\theta_{e \gamma}^{*}>10^{\circ}$. Systematic errors are estimated by variation of the cuts of Table 1 .

For comparison with previous experiments, the branching ratio with cuts $E_{\gamma}^{*}>10 \mathrm{MeV}, 0.6<$ $\cos \theta_{e \gamma}^{*}<0.9$ is

$$
R=\frac{B r\left(K^{-} \rightarrow \pi^{0} e^{-} \bar{v}_{e} \gamma\right)}{B r\left(K^{-} \rightarrow \pi^{0} e^{-} \bar{v}_{e}\right)}=(0.47 \pm 0.02(\text { stat. }) \pm 0.03(\text { syst. })) \times 10^{-2}
$$



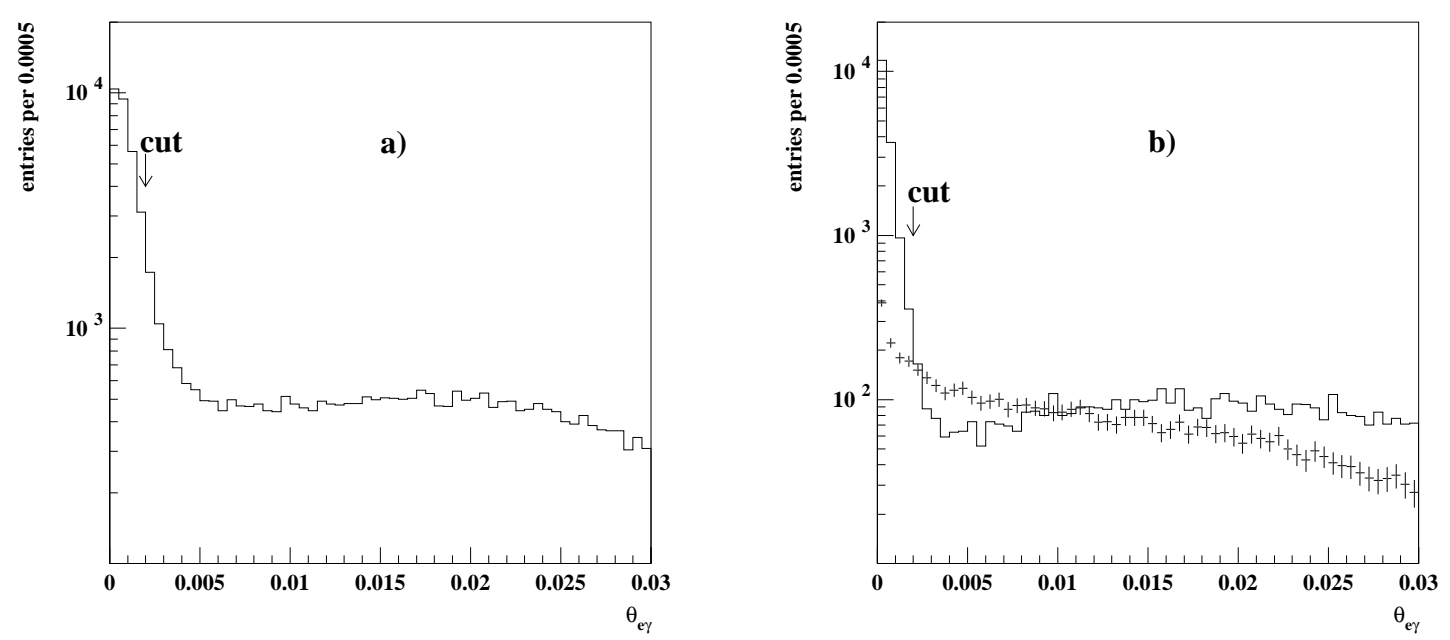

Figure 7: Distribution over $\theta_{e \gamma}$ - the angle between electron and photon in lab. system. a) Real data; b) MC background (histogram) and signal (points with errors).

\begin{tabular}{|c|c|c|}
\hline$R_{\text {exp }} \times 10^{2}$ & $N_{e v}$ & experiment \\
\hline $0.47 \pm 0.02$ & 1456 & this exp. \\
$0.46 \pm 0.08$ & 82 & XEBC [20] \\
$0.56 \pm 0.04$ & 192 & ISTRA [21] \\
$0.76 \pm 0.28$ & 13 & HLBC [22] \\
\hline
\end{tabular}

Table 2: $\operatorname{Br}\left(K^{-} \rightarrow \pi^{0} e^{-} \bar{v}_{e} \gamma\right) / \operatorname{Br}\left(K^{-} \rightarrow \pi^{0} e^{-} \bar{v}_{e}\right)$ for $E_{\gamma}^{*}>10 \mathrm{MeV}, 0.6<\cos \theta_{e \gamma}^{*}<0.9$ in comparison previous.

The results of previous experiments are given in Table 2 .

For the cuts $E_{\gamma}^{*}>30 \mathrm{MeV}$ and $\theta_{e \gamma}^{*}>20^{\circ}$, used in most theoretical papers, we obtain:

$$
R=\frac{B r\left(K^{-} \rightarrow \pi^{0} e^{-} \bar{v}_{e} \gamma\right)}{B r\left(K^{-} \rightarrow \pi^{0} e^{-} \bar{v}_{e}\right)}=(0.63 \pm 0.02(\text { stat. }) \pm 0.03(\text { syst. })) \times 10^{-2}
$$

Using new PDG[10] value for $K_{e 3}$ decay, the branching ratio for $K^{-} \rightarrow \pi^{0} e^{-} \bar{v}_{e} \gamma$ is:

$$
B r=(3.13 \pm 0.09 \pm 0.14) \times 10^{-4}
$$

It can be compared with theoretical prediction[3], at tree level, $\mathrm{Br}=2.8 \times 10^{-4}$ and at $O\left(p^{4}\right)$ in $\mathrm{ChPT}, \mathrm{Br}=3.0 \times 10^{-4}$. Theoretical prediction by V.V.Braguta, A.A.Likhoded, A.E.Chalov[11], at tree level, is $\mathrm{Br}=3.12 \times 10^{-4}$.

For the asymmetry $A_{\xi}$ (for the same cuts as in Table 2) we get

$$
A_{\xi}=-0.015 \pm 0.021
$$



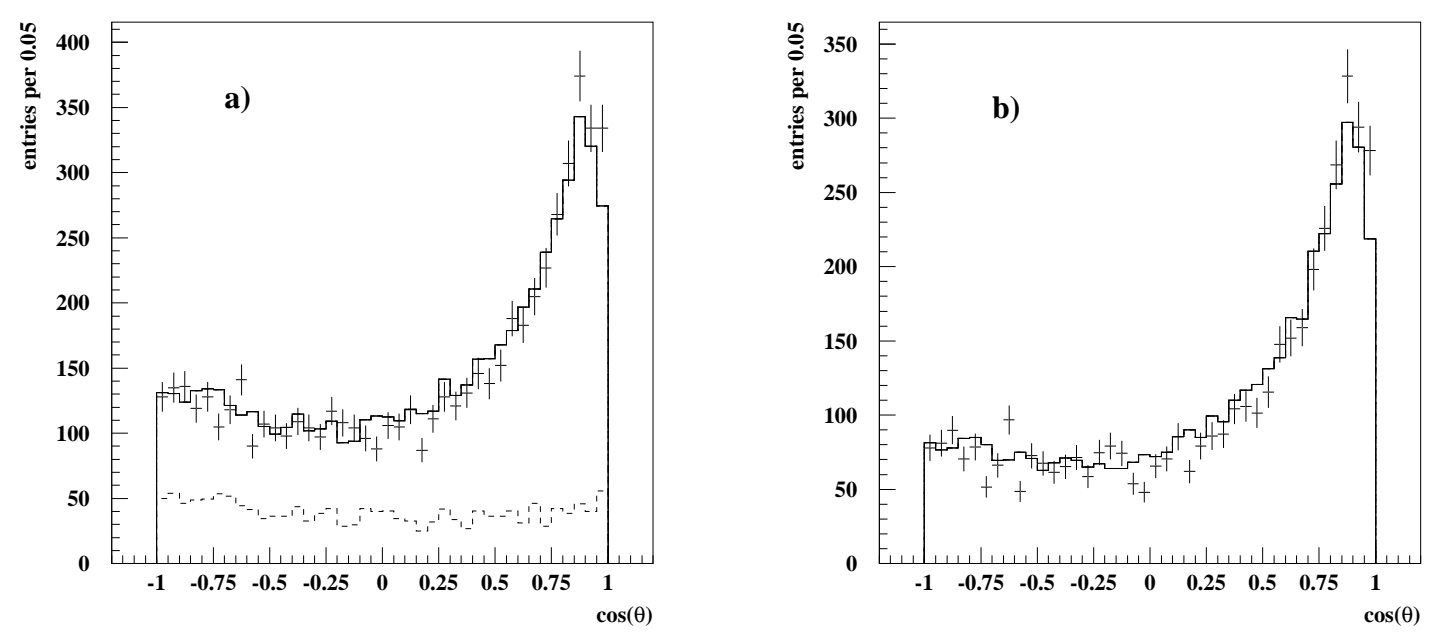

Figure 8: a) The distribution of the events over $\cos \theta_{e \gamma}^{*}$. Histogram is the real data, points with errors total MC signal plus background. Dotted line histogram is background. b) The same after background subtraction.

At present it is the best estimate of this asymmetry. It can be compared with an upper limit on the $A_{\xi}$ value $\left|A_{\xi}\left(K^{-} \rightarrow \pi^{0} e^{-} \bar{v}_{e} \gamma\right)\right|<0.8 \cdot 10^{-4}$ in the $S U(2)_{L} \times S U(2)_{R} \times U(1) \operatorname{model}[15]$ and $A_{\xi}=-0.59 \times 10^{-4}$ in the SM[1]].

\section{ACKNOWLEDGMENTS}

The authors would like to thank D.S. Gorbunov, V.A. Matveev and V.A. Rubakov, for numerous discussions and V.V.Braguta, A.A.Likhoded, A.E.Chalov for the program of matrix element calculation.

The work is supported in part by the RFBR grants 03-02-16330 (IHEP group), 06-02-16065a (INR group) and by Russian Science Support Foundation (INR group).

\section{References}

[1] J. Bijnens, G. Echer and J. Gasser, Nucl.Phys. B 396, (1993) 81.

[2] A. Pitch, Rep. Prog. Phys. 58, (1995) 563.

[3] L.Maiani, G.Pancheri and N.Paver, The Second DAFNE Physics Handbook (INFN-LNF-Divisione Ricerca, SIS-Ufficio Pubblicazioni, Frascati (Roma) Italy, ISBN 88-86409-02-8).

[4] J. Gasser et al.,hep-ph/0412130.

[5] N.Cabibbo, Phys.Rev.Lett., 10, (1963) 531.

[6] M. Kobayashiand, T. Maskawa, Progr.Theor. Phys. 49, (1973) 652.

[7] C. Jarlskog, Z.Phys. C29, (1985) 491. 


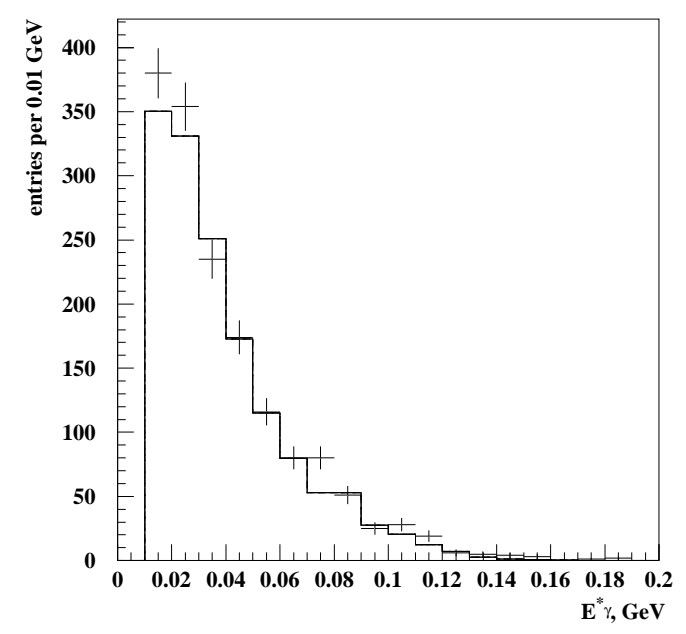

Figure 9: The distribution of the events over $E_{\gamma}^{*}$ the energy of the photon in the kaon rest frame. Histogram corresponds to the data, points with errors - total signal plus background.

[8] G.F. Farrar and M.E. Shaposhnikov, Phys.Rev.Lett., 70, (1993)2833, [Erratum , 71,(1993) 210][hep-ph/9305274]; P. Het and R. Sather, Phys.Rev. D 51,(1995) 379 [hep-ph/9404302]; M. Carena, M. Quiros and C.E.Wagner, Phys.Lett. B 380,(1996) 81. [hep-ph/9303420].

[9] J.Gevas, J.Iliopolus and J.Kaplan, Phys. Lett., 20, (1966) 432.

[10] W.-M. Yao et al. (Particle Data Group), J.Phys. G 65, (2006) 1.

[11] V.V. Braguta, A.A. Likhoded,and A.E. Chalov, Phys. Rev. D65, 054038 (2002) [hep-ph/0106147].

[12] Y. Kuzuruki Phys. Lett., B 193, (1987) 339.

[13] A. Bartl, T. Kernreiter and W. Porod, Phys Lett., B538,(2002) 59.

[14] N. Oshimo Mod. Phys. Lett., A4,(1989) 145.

[15] V.V. Braguta, A.A. Likhoded, A.E. Chalov, Phys.At.Nucl. 67,(2004) 1003, [Yad.Fiz. 67, (2004) 1025][hep-ph/0305067].

[16] V.N. Bolotov et al., IHEP preprint 8-98, 1998.

[17] R. Brun et al., Preprint CERN-DD/EE 84-1.

[18] I.V.Ajinenko et al., Yad. Fiz 67, (2002) 2125; I.V.Ajinenko et al., Phys. At. Nucl. 66, (2003) 2064; I.V.Ajinenko et al., Phys Lett., B 574, (2003) 14; O.P.Yuschenko et al., Phys Lett., B 589, (2004) 111.

[19] I.V.Ajinenko et al., Yad. Fiz. 66, (2003) 107; I.V.Ajinenko et al.,Phys. At. Nucl. 66, (2003) 105; O.P.Yuschenko et al., Phys Lett. B 581, (2004) 159.

[20] V.V.Barmin et al., SJNP 53, (1991) 606 , Yad. Fiz. 53, (1991)981.

[21] V.N.Bolotov et al., JETP Lett. 42, (1985) 481, Yad. Fiz. 44, (1986) 108.

[22] F.Romano et al., Phys Lett. 36B, (1971) 525. 